\title{
Dynamic Stability of a Composite Circular Cylindrical Shell Subjected to Combined Axial and Torsional Loading
}

\author{
A. ARGENTO \\ Department of Mechanical Engineering \\ University of Michigan-Dearborn \\ 4901 Evergreen Road \\ Dearborn, MI 48128-1491 \\ (Recelved April 6, 1992) \\ (Revised June 2, 1993)
}

\begin{abstract}
The dynamic stability of thin, clamped, composite circular cylindrical shells is studied for combined axial and torsional loading. Each load is taken to be harmonically varying; the frequencies of the two loads differ, in general. For the case in which the frequencies are commensurate, the applied load function is periodic. The equations of motion for the shell are reduced to a system of Hill equations by means of Fourier series expansions. Instability regions of principal and combination parametric resonance are determined by use of the monodromy matrix. Numerical results are generated for boron-epoxy layered shells for various cases of pure axial, pure torsional, and combined loading. The width of the principal instability region is presented as a function of fiber orientation for a laminate case. Stability diagrams are presented covering about 6 times the lowest natural frequency for various ratios of the applied axial and torsional frequencies.
\end{abstract}

KEY WORDS: dynamic stability, shell, composite, parametric resonance.

\section{INTRODUCTION}

A STRUCTURAL COMPONENT subjected to loads periodic in time may undergo an unstable dynamic response termed parametric resonance. This instability is considered dangerous because for a given load state it occurs over a range or ranges of forcing frequencies. In this article, the parametric resonance problem is studied for a layered composite circular cylindrical thin shell having clamped ends. The shell is subjected to a combination of harmonic axial and torsional loads having different frequencies.

A number of recent studies of the dynamic stability of composite plates are available; composite shells, however, have received less attention. Among the works on composite plates, References [1]-[5] are given, in which additional references are contained. Plates having general layering and subjected to harmonic 
undirectional loading are studied in Reference [1]. A nonlinear study, including damping, of antisymmetric cross-ply plates is given in Reference [2]. Studies of the effects of shear deformation are given in References [3]-[5]. In References [3] and [4], combinations of harmonic loads having the same frequency are considered. The dynamic stability of composite shells has been studied in References [6]-[9] for harmonic axial loading, and in References [10] and [11] for harmonic pressure loading. Parametric resonance induced by torsional loading alone has been studied for isotropic beam-type structures in Reference [12]. A study is given in Reference [13] of a general system of time dependent nonlinear equations containing simultaneous parametric and forcing (i.e., nonhomogeneous) excitations. Resonances involving combinations of the parametric and forcing excitations are considered.

Although work on parametric resonance of composite plates due to certain types of in-plane combined loading is available $[3,4]$, to the author's knowledge no analogous work exists for composite shells subjected simultaneously to axial and torsional loading. The previous work on shells [6]-[11] outlined above all treat various non-combined loading cases. In previous work by the author [7]-[9], pure axial loading is considered and the first order principal regions of parametric resonance are studied. In the present article, the load case treated in References [7]-[9] is extended to combined axial and torsional loading. The loads are permitted to have frequencies different from one another and the focus of the work is on the effects of combined loading and on several cases of distinct commensurate frequencies. Both principal and combination instability regions are studied.

The equations of motion are derived using linear Donnell type shell theory. Spatial dependence is satisfied by means of Fourier series expansions in the axial coordinate and a complex periodic function in the circumferential coordinate. If the load frequencies are commensurate, as assumed, this leads to a set of coupled Hill equations. For the case in which the two loads have common frequency, the Hill equations reduce to Mathieu equations. Numerical results are presented for the dynamic stability of boron-epoxy shells having various cases of antisymmetric layering and symmetric cross-ply layering.

\section{EQUATIONS OF MOTION}

The shell is taken to have length $2 l$, radius $a$ and is comprised of an arbitrary number of orthotropic layers. Displacement components of the shell's middle surface in the axial, circumferential, and radial directions are denoted by $u, v$, and $w$, respectively (see Figure 1). The shell is loaded by harmonic axial and torsional loads (per unit length), respectively given by

$$
\begin{aligned}
& P_{1}^{*}=\frac{1}{2 \pi a}\left(P_{o_{1}}+P_{t_{1}} \cos \Omega_{1} t\right) \\
& P_{2}^{*}=\frac{1}{2 \pi a}\left(P_{o_{2}}+P_{t_{2}} \cos \Omega_{2} t\right)
\end{aligned}
$$




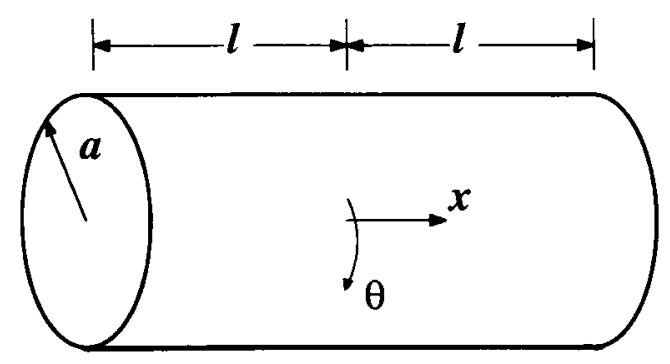

Figure 1. Beam geometry.

where $P_{o_{1}}$ and $P_{o_{2}}$ denote the static parts of the loads, $P_{t_{1}}$ and $P_{t_{2}}$ denote the amplitudes of the dynamic parts of the loads, $\Omega_{1}$ and $\Omega_{2}$ denote the frequencies of the dynamic loads, and $t$ denotes time.

As a matter of convenience, $P_{1}^{*}$ and $P_{2}^{*}$ are expressed in terms of the three quantities $P_{s}, P_{d}$ and $\Omega$ in the following way

$$
\begin{aligned}
& P_{o_{1}}=q_{o_{1}} P \\
& P_{o_{2}}=q_{o_{2}} P_{s} \\
& P_{t_{1}}=q_{d_{1}} P_{d} \\
& P_{t_{2}}=q_{d_{2}} P_{d} \\
& \Omega_{1}=m_{1} \Omega \\
& \Omega_{2}=m_{2} \Omega
\end{aligned}
$$

where the constants $q_{o_{1}}, q_{o_{2}}, q_{d_{1}}, q_{d_{2}}, m_{1}$, and $m_{2}$ are prescribed according to the particular load case.

The shell is modeled by linear Donnell theory. Classical lamination theory is used to determine the stiffness properties of the overall laminate shell structure. The equations governing the response of the shell are derived by applying Newton's law to a shell element in the deformed configuration. This approach, which is also used in static buckling theory, yields a set of coupled nonlinear partial differential equations. These are then linearized by the standard perturbation technique in which the response is separated into a pre-instability part, called the unperturbed response (subscript $p$ ) and an incremental perturbation part, called the perturbed response (subscript 1). The equations governing the perturbed response then become:

$$
D^{(1)} u_{1}+D^{(2)} v_{1}+D^{(3)} w_{1}=\bar{m} \frac{\partial^{2} u_{1}}{\partial t^{2}}
$$




$$
\begin{gathered}
D^{(2)} u_{1}+D^{(4)} v_{1}+D^{(5)} w_{1}=\bar{m} \frac{\partial^{2} v_{1}}{\partial t^{2}} \\
D^{(6)} u_{1}+D^{(7)} v_{1}+D^{(8)} w_{1}+2 N_{x \theta_{p}} \frac{\partial^{2} w_{1}}{\partial \theta \partial x}+\frac{1}{a} N_{\theta \theta_{p}} \frac{\partial^{2} w_{1}}{\partial \theta^{2}} \\
+a N_{x x_{p}} \frac{\partial^{2} w_{1}}{\partial x^{2}}+\bar{m} E_{43} \frac{\partial^{3} u_{1}}{\partial t^{2} \partial x}+\bar{m} E_{44} \frac{\partial^{3} v_{1}}{\partial t^{2} \partial x}=\bar{m} \frac{\partial^{2} w_{1}}{\partial t^{2}}
\end{gathered}
$$

where $D^{(1)}-D^{(8)}$ are differential operators given in the Appendix, $A_{\imath \jmath}, B_{\imath}$, and $D_{i j}$ are the usual laminate stiffness properties, and the $C_{t}$ and $E_{i}$ are constants given in Reference [8]. The quantities $N_{x \theta_{p}}, N_{x x_{p}}$, and $N_{\theta \theta_{p}}$ denote the unperturbed response stress resultants and are functions of the loads $P_{1}^{*}$ and $P_{2}^{*}$.

Solutions to Equations (3)-(5) are assumed in the form of Fourier series with respect to $x$ given by

$$
\begin{gathered}
\left(\begin{array}{c}
u_{1}(x, \theta, t) \\
v_{1}(x, \theta, t) \\
w_{1}(x, \theta, t)
\end{array}\right)= \\
\left\{\frac{1}{2}\left(\begin{array}{c}
A_{o}(t) \\
C_{o}(t) \\
f_{o}(t)
\end{array}\right)+\sum_{n=1}^{\infty}\left\{\left(\begin{array}{l}
A_{n}(t) \\
C_{n}(t) \\
f_{n}(t)
\end{array}\right) \cos \frac{n \pi x}{l}+\left(\begin{array}{c}
B_{n}(t) \\
D_{n}(t) \\
g_{n}(t)
\end{array}\right) \sin \frac{n \pi x}{l}\right)\right\} e^{t k \theta}
\end{gathered}
$$

where $k$ is the circumferential wave number, $i$ is the imaginary unit and the Fourier coefficients $A_{o}, A_{n}, B_{n}, C_{o}$, etc. are functions of time.

The shell is taken to have clamped supports. As is well known, there are four different types of clamping conditions. Here the following case is studied (commonly designated Case C 1, [14]).

$$
u_{1}=v_{1}=w_{1}=\frac{\partial w_{1}}{\partial x}=0, \quad x= \pm l
$$

The assumed solutions [Equation (6)] may be made to satisfy these boundary conditions through proper differentation of the Fourier series (see Reference [15]). Specific details can be found in Reference [8].

Substitution of Equation (6) and appropriate derivatives into Equations (3)-(5) leads to a system of time dependent ordinary differential equations having the following form.

$$
\underline{m} \frac{d^{2} \bar{f}}{d t^{2}}+\left(\underline{R}-P_{s} \underline{S}_{1}-P_{d} \underline{Q}(t)\right) \bar{f}=0
$$

where

$$
\underline{Q}(t)=\underline{S}_{2} \cos \left(m_{1} \Omega t\right)+\underline{S}_{3} \cos \left(m_{2} \Omega t\right)
$$


The quantities $\underline{m}, \underline{R}, \underline{S}_{1}, \underline{S}_{2}$, and $\underline{S}_{3}$ are constant matrices containing material and geometric properties, and $\bar{f}$ is the vector of unknowns. Note that equations reflecting the boundary conditions are also contained in Equations (8), see Reference [8].

For the case in which $m_{1}$ and $m_{2}$ are integers, the frequencies $\Omega_{1}$ and $\Omega_{2}$ are commensurate and so then the function $\underline{Q}(t)$ is periodic. The Equations (8) are then a system of Hill equations. If $m_{1}=m_{2}, Q(t)$ is harmonic and Equations (8) then form a system of Mathieu equations, which is a special case of Hill equations.

\section{STABILITY}

In this section, methods for determining the shell's dynamic stability will be reviewed.

In the case $m_{1}=m_{2}=1, \underline{Q}(t)$ is given by

$$
Q(t)=\left(\underline{S}_{2}+\underline{S}_{3}\right) \cos (\Omega t)
$$

The Equations (8) are then a system of Mathieu equations. For each circumferential wave number, $k$, the principal regions of parametric resonance emanate in load-frequency space from the values $\Omega=2 \omega_{i} / j$, where $\omega_{i}$ denote the natural frequencies for the particular $k$ value and $j$ is a positive nonzero integer. The regions associated with $j=1$ are termed the first order regions and are ordinarily the most significant of the principal regions. In addition, sum and difference combination resonances may occur. These emanate from the values $\Omega=$ $\left(\omega_{i}+\omega_{l}\right) / j$ and $\Omega=\left(\omega_{i}-\omega_{l}\right) / j$, respectively.

The principal regions of parametric resonance are most readily extracted from the system (8) (for $m_{1}=m_{2}$ ) by methods set forth in the text by Bolotin [16]. Specifically, the bounding branches of the instability regions are given by

$$
\operatorname{det}\left(\underline{R}-P_{o} \underline{S}_{1} \pm \frac{1}{2} P_{d}\left(\underline{S}_{2}+\underline{S}_{3}\right)-\frac{1}{4} \Omega^{2} \underline{m}\right)=0
$$

In addition, the natural frequencies of the statically loaded shell, are given by

$$
\operatorname{det}\left(\underline{R}-P_{o} \underline{S}_{1}-\omega^{2} \underline{m}\right)=0
$$

Here, the calculations (11) and (12) are made by conversion to the respective eigenvalue problems. Routines in the Eispack software library are used to perform the eigenvalue calculations.

The calculation (11) gives the principal regions of parametric resonance and only applies for $m_{1}=m_{2}$. In general, stability diagrams containing all possible regions of instability are determined using the monodromy matrix technique (see, for example, Reference [17]). To use this method, it is first assumed that $m_{1}$ and 
$m_{2}$ are integers so that the function $Q(t)$ in Equation (9) is periodic. The $n \times n$ system (8) is then cast as the $N \times N(N=2 n)$ first order equations:

$$
\frac{d \bar{Z}(t)}{d t}=\underline{A}(t) \bar{Z}(t)
$$

where $\bar{Z}$ is a vector containing $\bar{f}$ and $d \bar{f} / d t$, and $\underline{A}(t)$ is an $N \times N$ periodic matrix having period $T$.

Stability of the system (13) is determined by the eigenvalues of the so-called monodromy matrix, $\underline{M}$. This matrix may be obtained from the fundamental matrix of the system (13), $\Psi$, in the following way. Suppose that the $i$ th column of $\underline{\psi}(t)$ is generated by the special initial conditions

$$
\begin{gathered}
Z_{\jmath}=0: \quad j=1,2, \ldots, N \quad j \neq i \\
Z_{\iota}=1
\end{gathered}
$$

for each column $i=1,2, \ldots, N$. Then, it can be shown that $\underline{M}$ and $\underline{\psi}$ are related by

$$
\underline{M}=\underline{\psi}(T)
$$

According to Floquet's theorem, the system (13) is then stable so long as the eigenvalues of $\underline{M}$ have magnitude less than or equal to 1 .

The method is quite numerically intensive since the $N \times N$ system (13) must be numerically integrated $N$ times over interval $T$. Here a single pass scheme is adopted $[18,19]$ which results in reduced, although still high, computation time.

\section{NUMERICAL RESULTS}

Numerical results describing the stability of boron epoxy shells having fiber reinforced layers are presented in this section. The properties of the material are $\varrho=2048 \mathrm{~kg} / \mathrm{m}^{3}, E_{l} / E_{\mathrm{t}}=11.11, G_{l t} / E_{t}=.24, \nu_{l t}=.28$, where $\varrho$ denotes density, $E$ denotes Young's modulus, $G$ denotes shear modulus, $\nu$ denotes Poisson's ratio, and subscripts $l$ and $t$ denote the directions parallel and transverse to the fiber directions, respectively.

Figures 2-6 are for a shell consisting of three equal thickness layers and fiber orientations $-\theta / 0 / \theta$. The shell's geometry is $2 l / a=4$ and $a / h=400$ where $h$ is the total thickness of the shell.

To ensure that the shell is statically stable, the applied dynamic loads, $P_{1}^{*}$ and $P_{2}^{*}$, are chosen as fractions of the minimum static buckling loads. The normalized static buckling loads as a function of $\theta$ are given in Figures 2-4 for pure axial loading, pure torsional loading, and combined loading, respectively. In Figures 2 and 3 , the minimum critical buckling loads are normalized as

$$
\bar{P}_{1}=\frac{P_{o_{1}}^{\mathrm{crtt}}}{2 \pi a E_{t} h}
$$




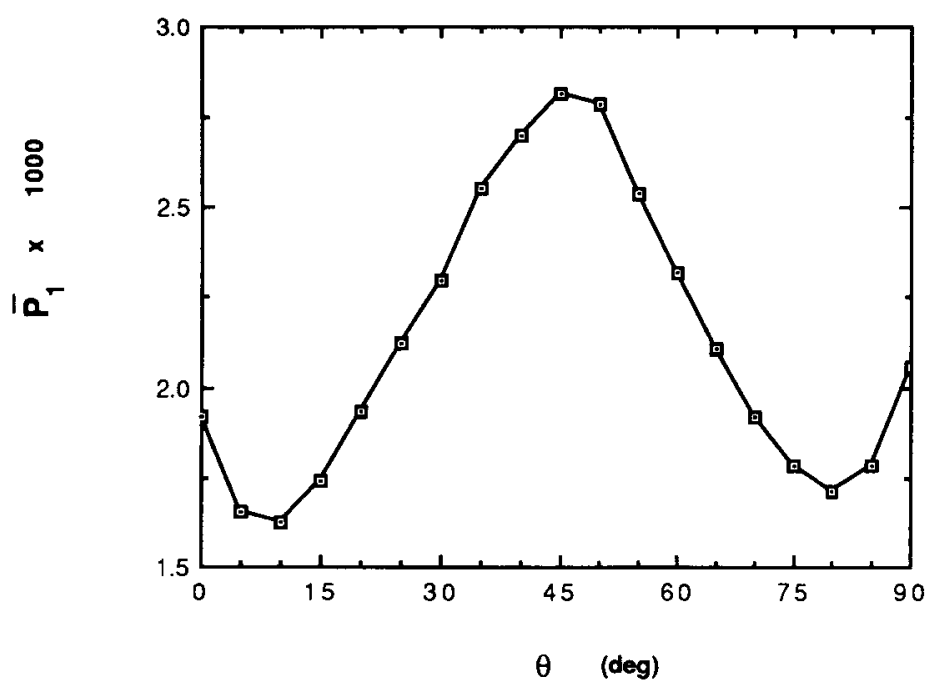

Figure 2. Minimum static buckling loads; pure axial loading: $\mathrm{q}_{\mathrm{o}_{1}}=1, \mathrm{q}_{\mathrm{o}_{2}}=\mathrm{q}_{\mathrm{d}_{1}}=\mathrm{q}_{\mathrm{d}_{2}}=0$.

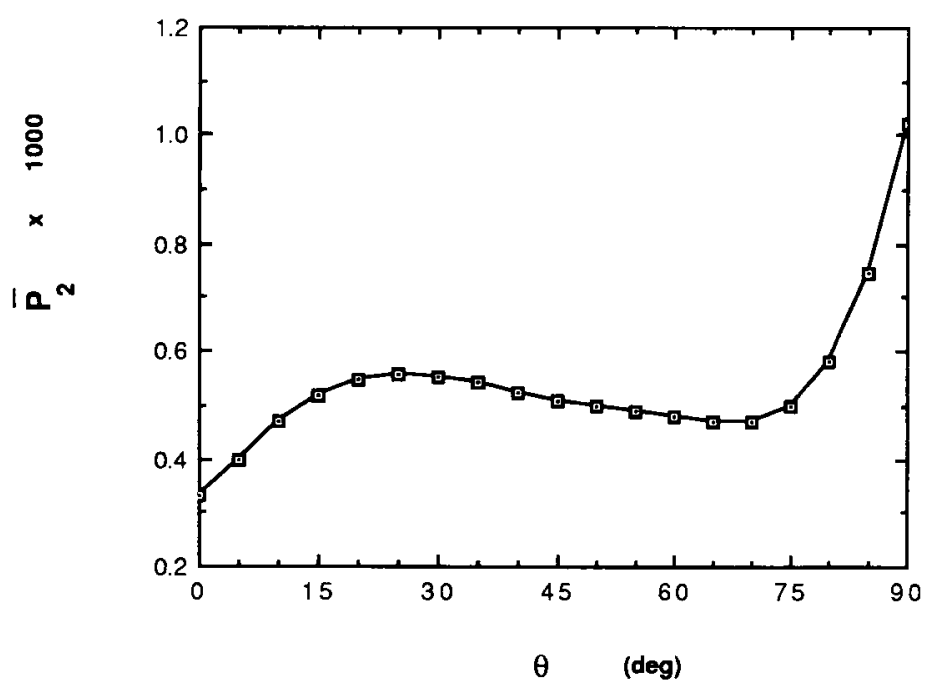

Figure 3. Minimum static buckling loads; pure torsional loading: $\mathrm{q}_{\mathrm{o}_{2}}=1, \mathrm{q}_{\mathrm{o}_{1}}=\mathrm{q}_{\mathrm{d}_{1}}=$ $\mathrm{q}_{\mathrm{d}_{2}}=0$. 


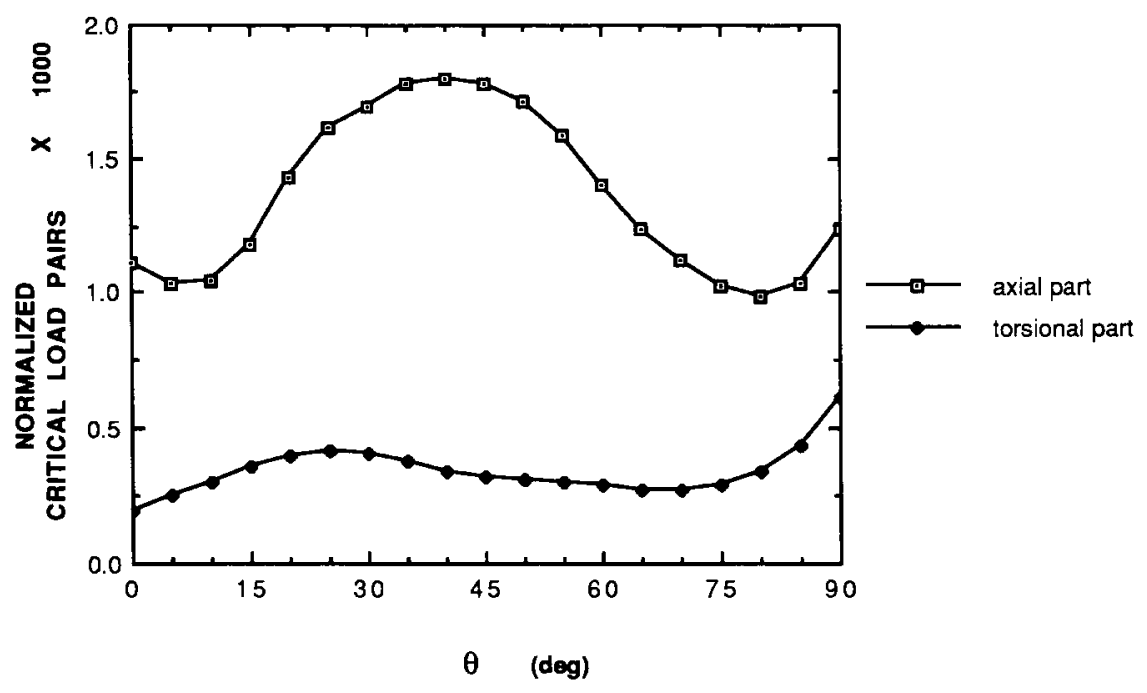

Figure 4. Combined loading static buckling load pairs.

and

$$
\bar{P}_{2}=\frac{P_{o_{2}}^{\mathrm{ran}}}{2 \pi a E_{t} h}
$$

$P_{o_{1}}^{\text {crit }}$ and $P_{o_{2}}^{\text {crit }}$ refer to the static buckling loads for the circumferential wave numbers which yield the minimum load values. In the combined loading case, the axial and torsional loads are taken to be applied in the ratio of their critical values when applied separately. Specifically, in Figure 4,

$$
\frac{q_{o_{2}}(\theta)}{q_{o_{1}}(\theta)}=\frac{\bar{P}_{2}(\theta)}{\bar{P}_{1}(\theta)}
$$

where $\bar{P}_{1}(\theta)$ and $\bar{P}_{2}(\theta)$ are the values from Figures 2 and 3, respectively. The critical value of $P_{s}$ is then calculated for loads applied in this ratio. The critical load pairs in Figure 4 are normalized as in the previous figures. Specifically,

$$
\begin{aligned}
& \bar{P}_{o_{1}}^{\mathrm{rIt \prime}}=\frac{q_{o_{1}} P_{s}^{\mathrm{crut}}}{2 \pi a E_{t} h} \\
& \bar{P}_{o_{2}}^{\mathrm{rrut}}=\frac{q_{o_{2}} P_{s}^{\mathrm{crut}}}{2 \pi a E_{t} h}
\end{aligned}
$$

In Figures 5 and 6, features of the first order principal region of parametric 


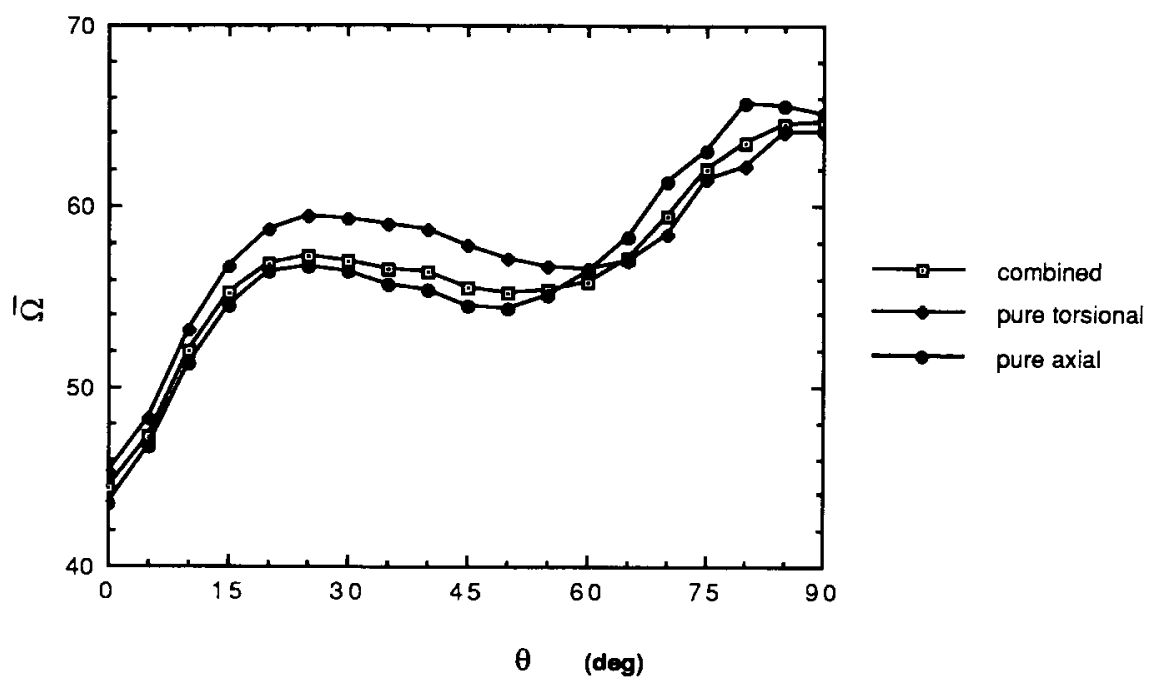

Figure 5. Frequency values on lower branch of first order principal regions of parametric resonance.

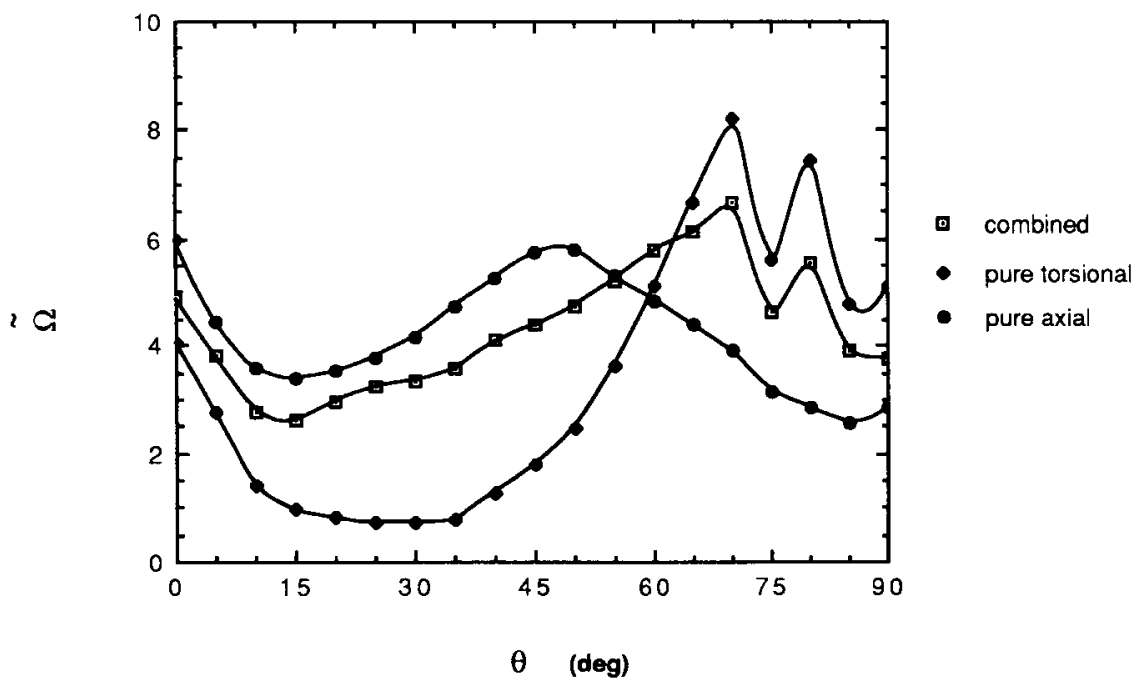

Figure 6. Relative widths of first order principal regions of parametric resonance. 
resonance are presented as a function of $\theta$ for the common frequency case, $m_{1}=m_{2}=1$. For the three load cases, Figure 5 gives the normalized lower branch parametric resonance frequency corresponding to $\omega_{1}$ and Figure 6 gives the corresponding normalized width of the region. The calculations are made by use of Equation (11). For each load case the shell is taken to be subjected to static and dynamic load magnitudes equal to .3 times the critical values given in Figures $2-4$. Thus, the shell is subjected to constant static loading and superimposed harmonic loading. The frequency values in Figure 5 are non-dimensionalized as in [6]:

$$
\bar{\Omega}=\left(\frac{\varrho}{E_{t}}\right)^{1 / 2} \frac{a^{2}}{h} \Omega
$$

In Figure $6, \tilde{\Omega}$ denotes the normalized relative widths of the instability regions. Denoting $\Omega^{u}$ as the lower branch value and $\Omega^{u}$ as the corresponding upper branch value, $\tilde{\Omega}$ is defined as:

$$
\tilde{\Omega}=\frac{\Omega^{u}-\Omega^{l}}{\Omega^{l}} \times 100
$$

The circumferential wave numbers, $k$, in Figures 5 and 6 are those which yield the lowest frequency regions. Table 1 gives the fiber orientations and the corresponding wave numbers for the three load cases.

In Figure 5, it is seen that the lower branch frequency value for each load case is quite affected by the fiber orientation. Likewise, in Figure 6, the relative widths of the instability regions are also quite affected by $\theta$. Also, the three curves in Figure 5 each vary in the same general way with $\theta$. However, in Figure 6 , the three relative width curves have somewhat different character from one another. This is especially apparent for $\theta=15^{\circ}$ to $\theta=35^{\circ}$ where the pure torsion region remains quite narrow, whereas the widths for the other two load cases are increasing.

Table 1. Critical circumferential wave numbers in Figures 5 and 6.

\begin{tabular}{cccc}
\hline $\boldsymbol{\theta}$ (deg.) & Axial & Torsional & Combined \\
\hline 0 & 7 & 8 & 7 \\
5 & 7 & 7 & 7 \\
$10-35$ & 8 & 8 & 8 \\
$40-70$ & 7 & 7 & 7 \\
75 & 6 & 7 & 6 \\
80 & 6 & 6 & 6 \\
$85-90$ & 5 & 5 & 5 \\
\hline
\end{tabular}


It should also be observed that although $\theta>60$ is a good choice of orientation if one wishes the instability frequencies to be relatively high, the widths of the pure torsion and combined load regions achieve their largest values in this range. Also, the pure axial buckling loads are relatively low for $\theta>60$. In general, overall analysis of the shell would be based on a number of factors including the expected forcing frequency range, the expected load magnitudes and whether torsion, axial and/or combined loading is expected.

In Figures 7-10, stability diagrams are presented for a 90/0/90 shell having $2 l / a=.5$ and $a / h=333.3$. The case of combined axial and torsional dynamic loading is studied. (The static parts of the loads are zero.). For this shell, the normalized minimum static buckling load pairs (combined load case) have been calculated to be $\bar{P}_{o_{1}}^{\text {cru }}=1.3356 \times 10^{-3}, \bar{P}_{o_{2}}^{\text {crt }}=.9616 \times 10^{-3}$ when the axial ar.d torsional loads are applied in the ratio of their critical values when applied separately. In this case the ratio is given by $q_{o_{1}}=1, q_{o_{2}}=.72$. The quantity $q$ in the figures defines the applied dynamic load magnitude and is given by the ratio $\bar{P}_{t_{1}} / \bar{P}_{o_{1}}^{\text {crit }}$ or $\bar{P}_{t_{2}} / \bar{P}_{o_{2}}^{\text {trut }}$. The minimum natural frequency was found using Equation (12) to occur at $k=9$. The first three normalized natural frequencies for $k=9$ are $\bar{\omega}_{1}=147.9, \bar{\omega}_{2}=301.6$ and $\bar{\omega}_{3}=396.9$. The instability of the shell, for $k=9$, in the frequency range covering approximately 6 times its lowest natural frequency is studied in the figures for the following frequency cases: $m_{1}=m_{2}=1 ; m_{1}=1, m_{2}=2 ; m_{1}=2, m_{2}=3 ; m_{1}=3, m_{2}=2$. The results in these figures were generated using the monodromy matrix technique described in the previous section. Load-frequency space was swept using a frequency iteration value of $\Delta \bar{\Omega}=.45$. More exact location of the instability regions was determined by refinement in the vicinity of the branches. Very narrow instability regions having widths less than about .45 are not shown since damping in the system would most likely prevent them from being physically realized. The principal regions of parametric resonance can also be determined by Equation (11) for the $m_{1}=m_{2}=1$ case and so was used as a check. It was found that Equation (11) gave principal regions nearly identical to those shown in Figure 7. In all four figures it can be seen that only principal and sum combination resonances occur for this shell; difference combination resonances did not occur. Also, only sum combination resonances corresponding to odd-even frequency mode pairs occurred. In all cases, the instability regions were bounded by almost exactly straight lines.

In Figure 7 the common frequency case is studied. There it is seen that the widest regions are those of principal parametric resonance corresponding to the second and third natural frequencies. The first (lowest frequency) combination resonance is very narrow but the second combination resonance is about twice as wide as the lowest principal region. The normalized frequency range from 600 to 800 is more dangerous from the standpoint of parametric resonance than the lower frequency range.

In Figure 8, a case in which the torsional loading is applied at twice the frequency of the axial loading is given. The left vertical scale gives the frequency of the axial loading, and the right vertical scale gives the frequency of the torsional loading. It is seen that principal parametric resonance occurs when the 


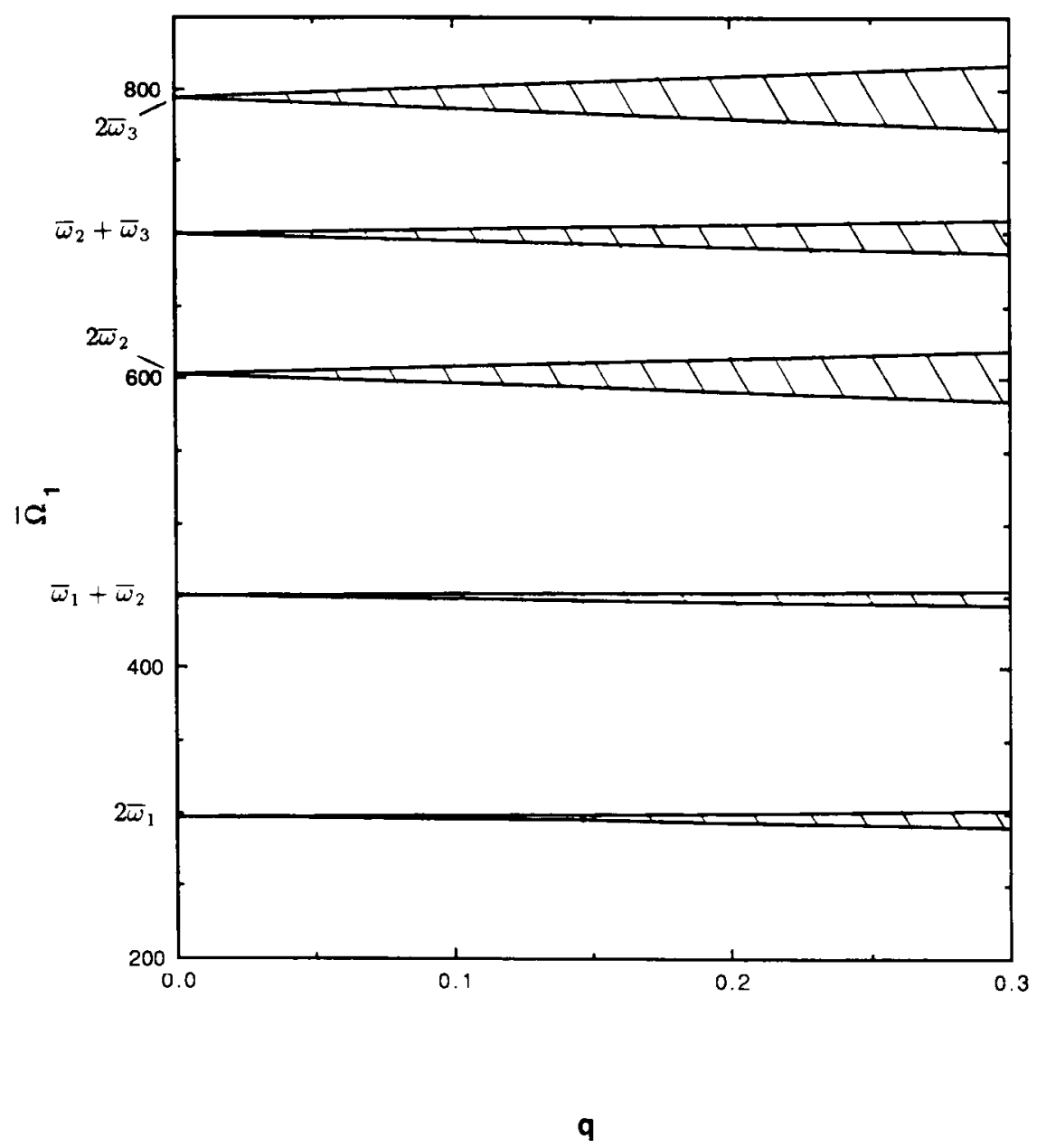

Figure 7. Stability diagram, combined loading, $m_{1}=m_{2}=1$. 


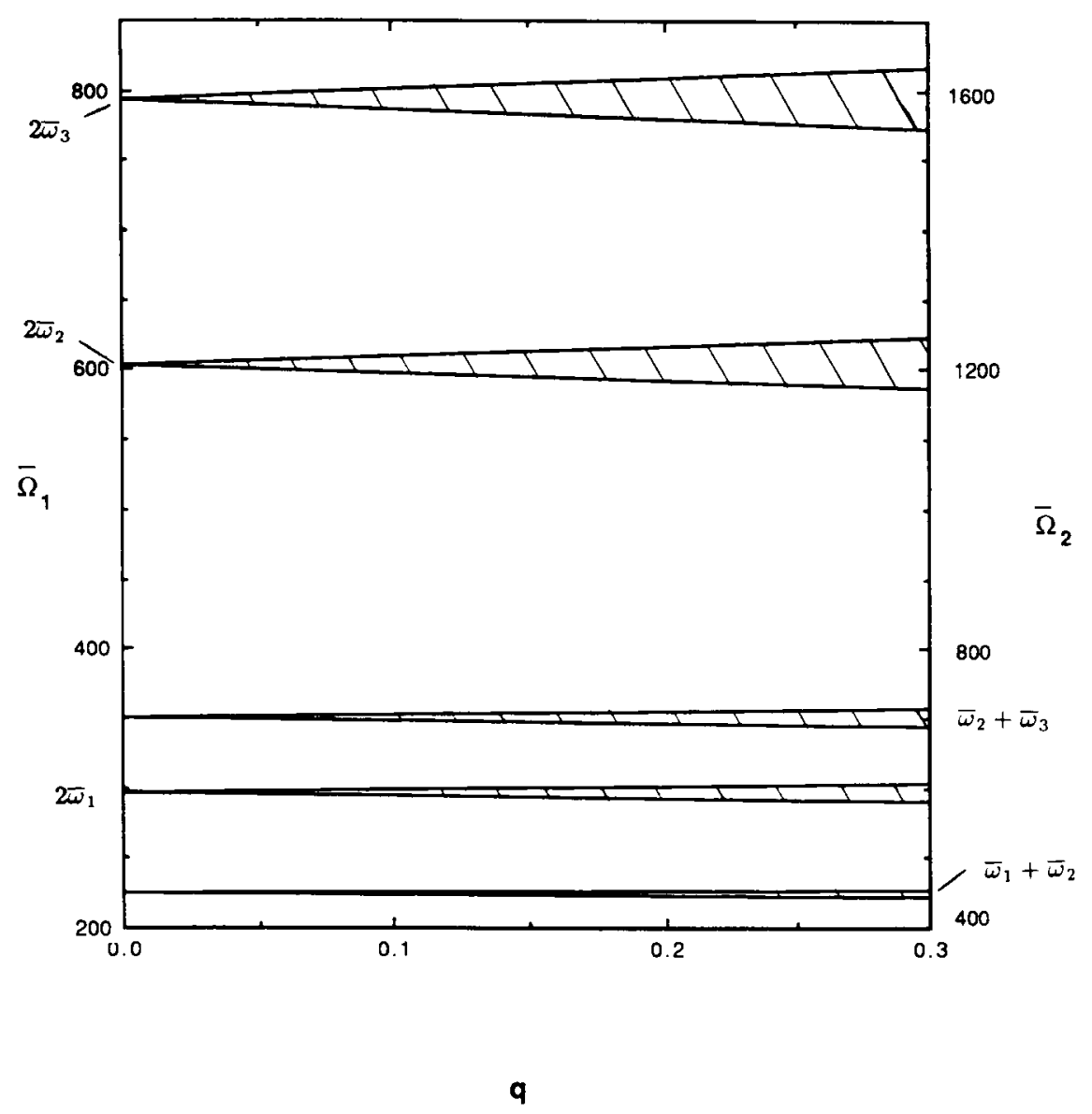

Figure 8. Stability diagram, combined loading, $\mathrm{m}_{1}=1, \mathrm{~m}_{2}=2$. 
axial loading frequency is near $2 \bar{\omega}_{1}, 2 \bar{\omega}_{2}$, and $2 \bar{\omega}_{3}$, and sum combination resonances occur when the torsional loading frequency is near $\bar{\omega}_{1}+\bar{\omega}_{2}$ and $\bar{\omega}_{2}+$ $\bar{\omega}_{3}$. The widths of the principal regions are about the same as in Figure 7 , but the combination regions are less wide than in Figure 7. Although the combination regions are fairly narrow, in this frequency case they cluster near the $2 \bar{\omega}_{1}$ region to cause a relatively unstable range of load frequency space.

In Figures 9 and 10 two additional frequency cases are presented, $m_{1}=2$, $m_{2}=3$ and $m_{1}=3, m_{2}=2$, respectively. In both these cases the $\bar{\omega}_{1}+\bar{\omega}_{2}$ region becomes very narrow and so is not shown. Also, as in Figure 8, the principal regions occur at the appropriate axial loading frequencies, and the combination resonance occurs at the appropriate torsional loading frequency. Comparing to the common frequency case, Figure 7, all the regions in Figures 9 and 10 are less

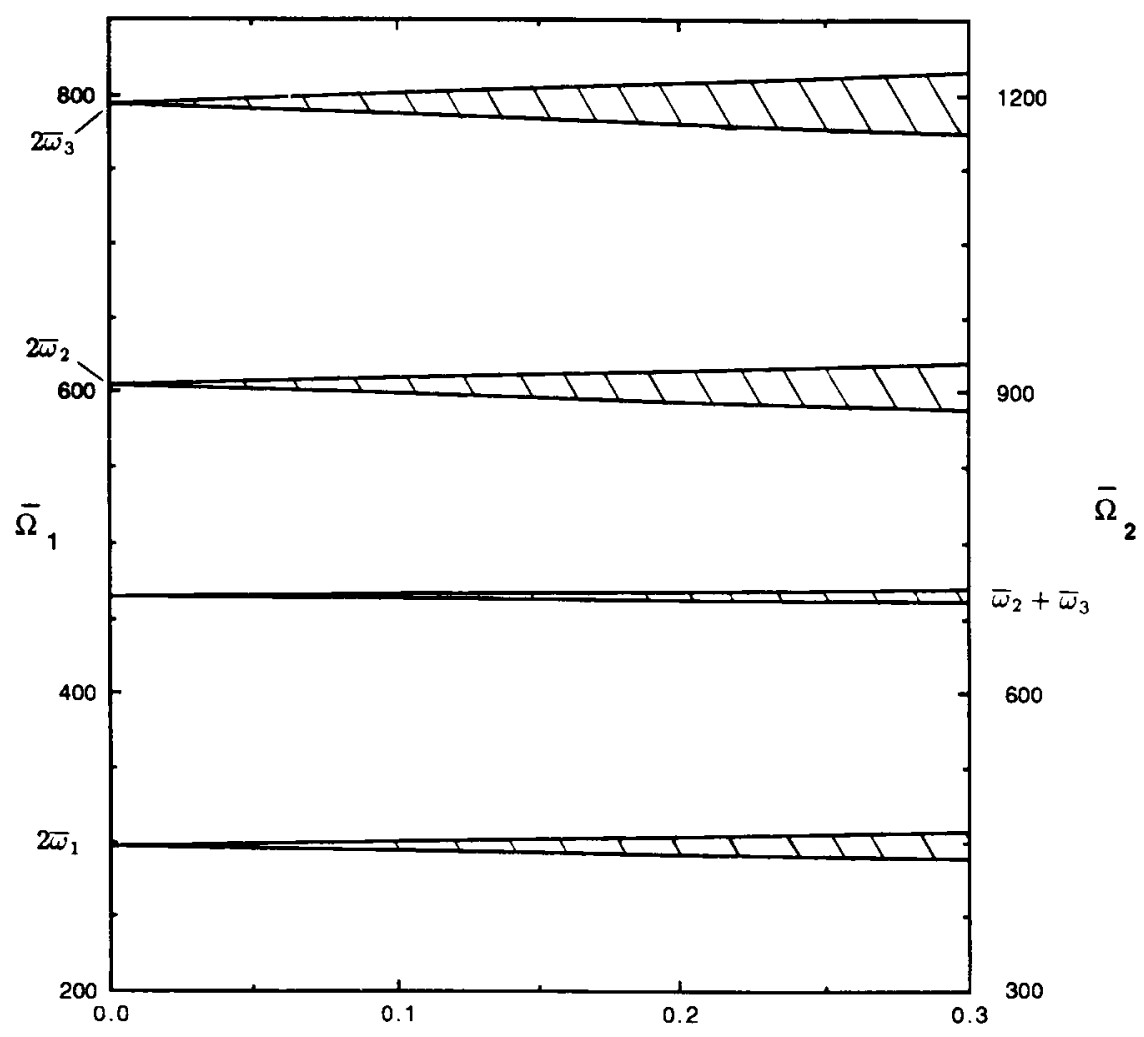

q

Figure 9. Stability diagram, combined loading, $m_{1}=2, m_{2}=3$. 


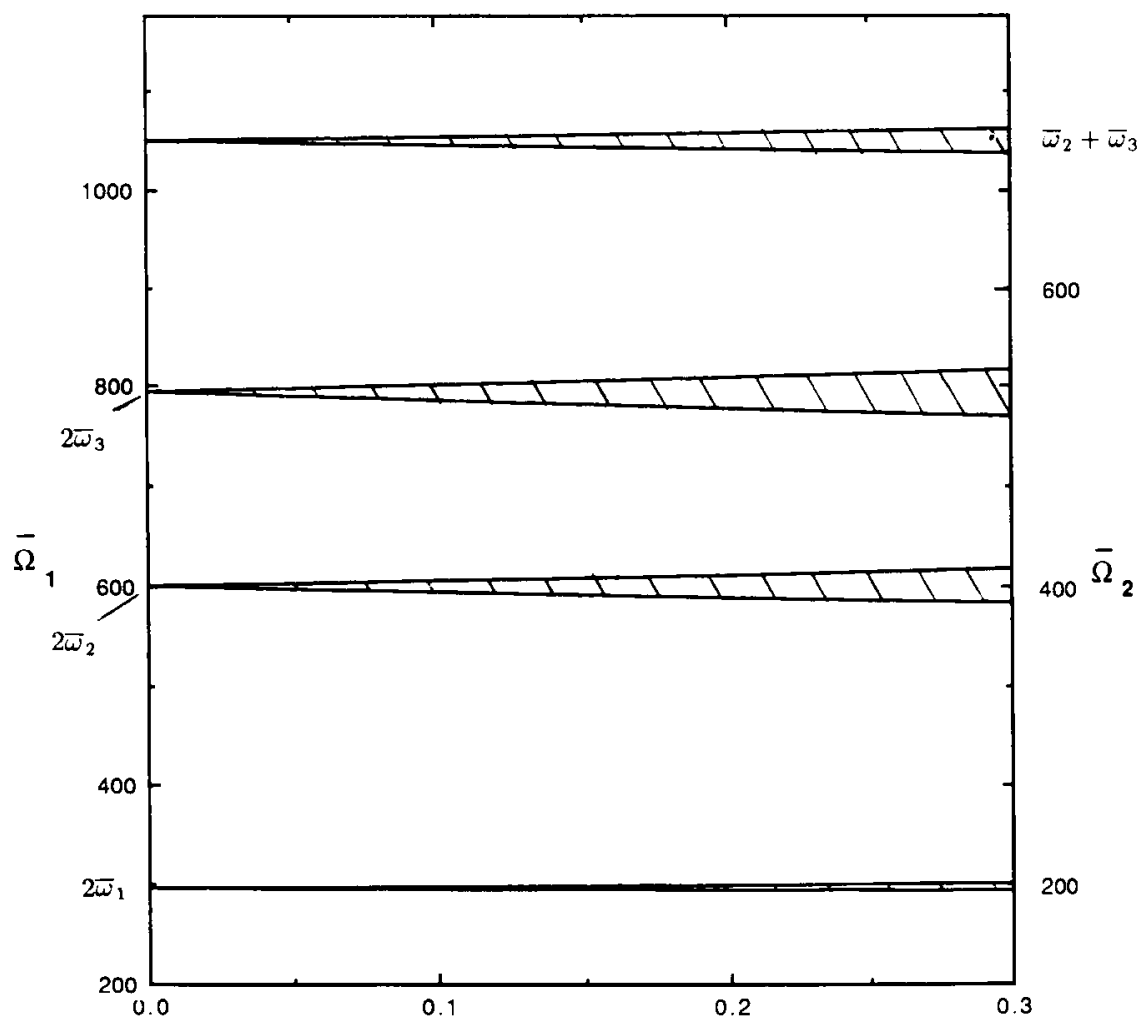

$\mathbf{q}$

Figure 10. Stability diagram, combined loading, $m_{1}=3, m_{2}=2$.

wide than their counterparts in Figure 7 except the $2 \bar{\omega}_{1}$ region in Figure 9 . Also, the instability regions do not tend to cluster as in Figure 8. Roughly, it can be said that the $m_{1}=2, m_{2}=3$ and $m_{1}=3, m_{2}=2$ frequency cases would be less likely to induce instability than the prior two cases.

\section{CONCLUSIONS}

The dynamic stability of clamped composite shells subjected to combined harmonic axial and torsional loading has been studied. Plots were given describing the effect of fiber orientation on the lowest first order region of principal parametric resonance. Stability diagrams covering about 6 times the lowest natural frequency have been constructed for various load cases. Principal and sum combina- 
tion resonances have been found to occur for the cases studied here. It has been shown that the widths of the instability regions and the relative location of the regions in load frequency space is affected by the ratio of the frequencies of the applied loads.

\section{APPENDIX}

$$
\begin{aligned}
D^{(1)} & =a A_{11} \frac{\partial^{2}}{\partial x^{2}}+\frac{A_{44}}{a} \frac{\partial^{2}}{\partial \theta^{2}}+2 A_{14} \frac{\partial^{2}}{\partial \theta \partial x} \\
D^{(2)} & =C_{1} \frac{\partial^{2}}{\partial x^{2}}+\frac{C_{5}}{a} \frac{\partial^{2}}{\partial \theta^{2}}+E_{1} \frac{\partial^{2}}{\partial \theta \partial x} \\
D^{(3)} & =-a B_{11} \frac{\partial^{3}}{\partial x^{3}}-A_{12} \frac{\partial}{\partial x}-\frac{B_{24}}{a^{2}} \frac{\partial^{3}}{\partial \theta^{3}}-\frac{A_{24}}{a} \frac{\partial}{\partial \theta} \\
& +E_{2} \frac{\partial^{3}}{\partial \theta^{2} \partial x}-3 B_{14} \frac{\partial^{3}}{\partial \theta \partial x^{2}} \\
D^{(4)} & =C_{3} \frac{\partial^{2}}{\partial x^{2}}+E_{6} \frac{\partial^{2}}{\partial \theta^{2}}+E_{3} \frac{\partial^{2}}{\partial \theta \partial x} \\
D^{(5)} & =-C_{4} \frac{\partial^{3}}{\partial x^{3}}-C_{5} \frac{\partial}{\partial x}+E_{8} \frac{\partial^{3}}{\partial \theta^{3}}+E_{7} \frac{\partial}{\partial \theta}+E_{5} \frac{\partial^{3}}{\partial \theta \partial x^{2}}+E_{4} \frac{\partial^{3}}{\partial \theta^{2} \partial x} \\
D^{(6)} & =E_{39} \frac{\partial^{3}}{\partial \theta \partial x^{2}}+E_{35} \frac{\partial^{3}}{\partial \theta^{2} \partial x}+A_{12} \frac{\partial}{\partial x}+\frac{B_{24}}{a^{2}} \frac{\partial^{3}}{\partial \theta^{3}}+\frac{A_{24}}{a} \frac{\partial}{\partial \theta} \\
& +E_{42} \frac{\partial^{4}}{\partial \theta \partial x^{3}}-C_{8}-\frac{D_{22}}{a^{3}} \frac{\partial^{4}}{\partial \theta^{4}}-2 \frac{B_{22}}{a^{2}} \frac{\partial^{2}}{\partial \theta^{2}} \\
D^{(8)} & =E_{40} \frac{\partial^{3}}{\partial \theta \partial x^{2}}+E_{36} \frac{\partial^{3}}{\partial \theta^{2} \partial x}+C_{5} \frac{\partial}{\partial x}+E_{8} \frac{\partial^{3}}{\partial \theta^{3}}-E_{7} \frac{\partial}{\partial \theta} \\
& =E_{33} \frac{\partial^{4}}{\partial x^{4}}+E_{34} \frac{\partial^{2}}{\partial x^{2}}+E_{37} \frac{\partial^{4}}{\partial \theta^{3} \partial x}+E_{38} \frac{\partial^{2}}{\partial \theta \partial x}+E_{41} \frac{\partial^{4}}{\partial \theta^{2} \partial x^{2}}
\end{aligned}
$$

\section{REFERENCES}

1. Srinivasan, R. S. and P. Chellapandi. 1986. "Dynamic Stability of Rectangular Laminated Composite Plates," Computers and Structures, 24:233-238.

2. Tylikowski, A. 1989. "Dynamic Stability of Nonlinear Antisymmetrically-Laminated Cross-Ply Rectangular Plates," American Society of Mechanical Engineers Journal of Applied Mechanics, 56:375-381.

3. Librescu, L. and S. Thangiitham. 1990. "Parametric Instability of Laminated Composite Shear- 
Deformable Flat Panels Subjected to In-Plane Edge Loads." Int. J. Non-Linear Mechanics, 25:263-273.

4. Bert, C. W. and V. Birman. 1987. "Dynamic Instability of Shear Deformable Antisymmetric Angle-Ply Plates," Int. J. Solids Structures, 23:1053-1061.

5. Moorthy, J., J. N. Reddy and R. H. Plaut. 1990. "Parametric Instability of Laminated Composite Plates with Transverse Shear Deformation," Int. J. Solids Structures, 26:801-811.

6. Bert, C. W. and V. Birman. 1988. "Parametric Instability of Thick Orthotropic, Circular Cylindrical Shells," Acta Mechanica, 71:61-76.

7. Argento, A. and R. A. Scott. 1989. "Parametric Resonance of Arbitrarily Layered Fiber Reinforced Composite Circular Cylindrical Shells," Proceedings of the 4th Technical Conference of the American Society of Composites, pp. 649-656.

8. Argento, A. and R. A. Scott. 1993. "Dynamic Instability of Layered Anisotropic Circular Cylindrical Shells, Part I: Theoretical Development," Journal of Sound and Vibration, 162:311-322.

9. Argento, A. and R. A. Scott. 1993. "Dynamic Instability of Layered Anisotropic Circular Cylindrical Shells, Part 2: Numerical Results," Journal of Sound and Vibration, 162:323-332.

10. Goroshko, O. A. and V. V. Emel'yanenko. 1975. "Dynamic Stability of Layered Anisotropic Shells," Soviet Applied Mechanics, UDC539.3:534.1, pp. 720-725.

11. Stuart, R. J., S. Dharmarajan and L. E. Penzes. 1980. "Dynamic Stability of Fibrous Composite Cylinders," in Fibrous Composites in Structural Design. New York: Plenum Press, pp. 329-340.

12. Aida, T. 1989. "Dynamic Stability of Thin-Walled Structural Members under Periodic Axial Torque," ASCE Journal of Engineering Mechanics, 115:71-88.

13. Plaut, R. H., J. J. Gentry and D. T. Mook. 1990. "Non-Linear Structural Vibrations under Combined Multi-Frequency Parametric and External Excitations," Journal of Sound and Vibration, 140:381-390.

14. Brush, D. O. and B. O. Almroth. 1975. Buckling of Bars, Plates, and Shells. New York: McGrawHill.

15. Whitney, J. M. and C. T. Sun. 1975. "Buckling of Composite Cylindrical Characterization Specimens," J. Composite Materials, 9:138-148.

16. Bolotin, V. V. 1964. The Dynamic Stability of Elastic Systems. San Francisco: Holden-Day.

17. Meirovitch, L. 1970. Methods of Analytical Dynamics. New York: McGraw-Hill.

18. von Kerczek, C. and S. H. Davis. 1975. "Calculation of Transition Matrices," AIAA Journal, 13:1400-1403.

19. Gaonkar, G. H., D. S. Simha Prasad and D. Sastry. 1981. "On Computing Floquet Transition Matrices of Rotorcraft," Journal of the American Helicopter Society, 26:56-61. 